\title{
ON A THEOREM OF MENCHOFF
}

\author{
Y. KATZNELSON
}

ABSTRACT. Construction of a continuous real-valued function which disagrees almost everywhere with any absolutely convergent Fourier series.

A classical theorem of Menchoff states that if $f$ is a finite measurable function on the circle $T$ and if $\epsilon>0$, there exist functions $g$ on $T$ with uniformly convergent Fourier series and such that $f(t)=g(t)$ except possibly on a set of measure less than $\epsilon$. The question has been raised (e.g. [1, problem 7, p. 527]) whether the theorem can be strengthened to yield, for any $f$ and $\epsilon$ as above, an absolutely convergent Fourier series whose sum $g(t)$ agrees with $f(t)$ except for a set of $t$ of measure less than $\epsilon_{\text {. Our purpose }}$ in this note is to show that this cannot be done; in fact we prove more:

Theorem. There exists a continuous real-valued function $f$ on $T$ such that if $g$ is the sum of an absolutely convergent Fourier series then $\{t ; f(t)=$ $g(t)\}$ has measure zero.

One can sharpen the problem by imposing on $f$ a modulus of continuity; in fact one may conjecture that there exists an $f \in \operatorname{Lip} 1 / 2$ which differs from any absolutely convergent Fourier series almost everywhere. The construction we give below yields a function whose modulus of continuity is bounded by $(-\log h)^{-1}$.

We denote by $A(T)$ the Banach algebra of absolutely convergent Fourier series. For a closed set $E \subset T$, we denote by $A(E)$ the Banach algebra of restrictions to $E$ of the elements of $A(T)$, the norm being the standard (quotient) norm.

The basic tool is the so-called Rudin-Shapiro sequence $\left\{\epsilon_{n}\right\}, \epsilon_{n}= \pm 1$, $n=0,1,2, \ldots$, which has the property that for all positive integers $N, M$

$$
\left\|\sum_{N+1}^{N+M} \epsilon_{n} e^{i n t}\right\|_{C(T)} \leq 5 \sqrt{M}
$$

(cf. [2, pp. 33-34]).

Define for $x \geq 0$,

$$
\Phi(x)= \begin{cases}\epsilon & n \leq x \leq n+1 / 2, \\ \text { linear } \text { for } n+1 / 2 \leq x \leq n+1,\end{cases}
$$

Received by the editors October 18, 1974.

AMS (MOS) subject classifications (1970). Primary 42A28.

Copyright $\odot$ 1975. American Mathematical Society 


$$
\phi_{k}(t)=\Phi\left(8^{k} t / 2 \pi\right), \quad 0 \leq t \leq 2 \pi .
$$

One checks in the construction of $\left\{\epsilon_{n}\right\}$ that $\epsilon_{0}=\epsilon_{8 k}=1$ for all $k$ and, thus, $\phi_{k}(0)=\phi_{k}(2 \pi)=1$, and we continue $\phi_{k}$ by periodicity or consider it as a function on $T=R / 2 \pi Z$.

We now write $k_{m}=m$ ! and $a_{m}=k_{m}^{-1}$, notice that

$$
a_{m}^{-1} 2^{3 k-1}{ }^{-k}=o(1), \quad a_{m}^{-1} a_{m+1}=o(1), \quad a_{m} k_{m}=1
$$

and claim that

$$
f=\sum a_{m} \phi_{k_{m}}
$$

satisfies the condition imposed in the theorem. The proof uses the following simple

Lemma. Let $k$ be a positive integer, $l$ and $n$ positive integers such that $l+n \leq 8^{k}$. Assume that $g \in C(T)$ and for some real-valued $c$ and some $\alpha, 0 \leq a \leq 1 / 2$, we have

$$
\left|g(t)-\phi_{k}(t)-c\right|<1 / 10
$$

on $90 \%$ of the points of $E$ where $E=\left\{2 \pi(a+j) 8^{-k}\right\}_{j=l}^{l+n}$. Then $\|g\|_{A(E)}>$ $\pi^{-1} \log n-4$.

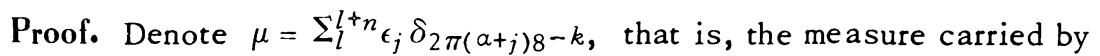
$E$ whose mass at $2 \pi(\alpha+j) 8^{-k}$ is equal to $\epsilon_{j}$. By (1) we have $\|\hat{\mu}\|_{\infty} \leq 5 \mathrm{~V} n$.

Select a real number $c_{1}$ so that $c_{1}=c(\bmod 4)$ and $\left|c_{1}\right| \leqq 2$. By (6), we have

Thus,

$$
\int \sin \frac{\pi}{2}\left(\operatorname{Re} g-c_{1}\right) d \mu>\frac{7}{10} n
$$

$$
\frac{7}{10} n \leq\left\|\sin \frac{\pi}{2}\left(\operatorname{Re} g-c_{1}\right)\right\|_{A(E)}\|\hat{\mu}\|_{\infty} \leq 5 \sqrt{n}\left\|\sin \frac{\pi}{2}\left(\operatorname{Re} g-c_{1}\right)\right\|_{A(E)},
$$

and

$$
\frac{7}{50} \sqrt{ } n \leq\left\|\sin \frac{\pi}{2}\left(\operatorname{Re} g-c_{1}\right)\right\|_{A(E)} \Rightarrow\|g\|_{A(E)} \geq \frac{2}{\pi} \log \frac{7 \sqrt{ } n}{50}-\left|c_{1}\right|,
$$

that is

$$
\|g\|_{A(E)} \geq \pi^{-1} \log n-\left(2+2 \pi^{-1} \log (50 / 7)\right)
$$

and the lemma follows.

We now turn to the proof that the function $f$ defined by (5) satisfies the condition stated in the theorem.

Let $g \in A(T)$ and assume that $G=\{t ; g(t)=f(t)\}$ has positive measure. Let $t_{0}$ be a point of density of $G$. For all sufficiently large $m$, the relative 
measure of $G$ in $I_{m}=\left(t_{0}-2 \pi 2^{-k_{m}}, t_{0}+2 \pi 2^{-k_{m}}\right)$ is greater than $99 \%$.

It is clear that for an appropriate $a_{m}, 0 \leq a_{m} \leq 1 / 2, G$ contains at least $98 \%$ of the points of the form $2 \pi\left(\alpha_{m}+j\right) 8^{-k_{m}}$ which are contained in $I_{m}$. We denote the set of all these points by $E_{m}$. $E_{m}$ is an arithmetical progression of length $2 \cdot 4^{k_{m}}$.

We now write

$$
f=\sum_{1}^{m-1} a_{j} \phi_{k_{j}}+a_{m} \phi_{k_{m}}+\sum_{m+1}^{\infty} a_{j} \phi_{k_{j}}
$$

and notice that the first sum differs from a constant on $I_{m}$ by at most $2^{2-k_{m}}$ $\Sigma_{1}^{m-1} a_{j} 8^{k_{j}}<<2^{3 k_{m}-1-k_{m}}$ (the half length of $I_{m}$ being $2 \pi 2^{-k_{m}}$ and the maximum slope of the sum is bounded by $(2 \pi)^{-1} 4 \Sigma_{1}^{m-1} a_{j} 8^{k} j$.

On the other hand, $\Sigma_{m+1}^{\infty} a_{j} \phi_{k_{j}}$ is uniformly bounded by $2 a_{m+1}$. Thus

$$
a_{m}^{-1}\left(g-g\left(t_{0}\right)\right)=\phi_{k_{m}}+\text { const }+o(1)
$$

on more than $90 \%$ of the points of $E_{m}$ and it follows by the lemma that

and so

$$
\left\|a_{m}^{-1}\left(g-g\left(t_{0}\right)\right)\right\|_{A\left(E_{m}\right)} \geq \pi^{-1} k_{m}
$$

$$
\left\|g-g\left(t_{0}\right)\right\|_{A\left(I_{m}\right)}>\pi^{-1} a_{m} k_{m}=\pi^{-1} .
$$

On the other hand, a classical theorem of Wiener $[3$, p. 88] implies that $\left\|g-g\left(t_{0}\right)\right\|_{A\left(I_{m}\right)} \rightarrow 0$ when $\left\{I_{m}\right\}$ are neighbourhoods of $t_{0}$ which shrink to $t_{0}$. This contradiction concludes the proof.

\section{B IB LIOGR A PHY}

1. N. K. Bari, A treatise on trigonometric series, Fizmatgiz, Moscow, 1961; English transl., Macmillan, New York; Pergamon Press, Oxford, 1964. MR 23 \#A3411; $30 \# 1347$.

2. Y. Katznelson, An introduction to harmonic analysis, Wiley, New York, 1968. MR $40 \# 1734$.

3. N. Wiener, The Fourier integral and certain of its applications, Cambridge Univ. Press, Cambridge, 1933; reprint, Dover, New York, 1959. MR 20 \#6634.

DEPARTMENT OF MATHEMATICS, HEBREW UNIVERSITY, JERUSALEM, ISRAEL 